\title{
Integração e Envolvimento dos participantes em MOOCs: contributo do
}

\section{BootCamp}

\section{Participants Integration and Engagement in MOOCs: BootCamp contribution}

\author{
Diana Morais, Lina Morgado \\ Universidade Aberta, Portugal
}

\begin{abstract}
Resumo
Esta comunicação pretende analisar o impacto da vivência dos primeiros dias online pelos participantes em contextos abertos e massivos - Cursos Abertos Online e Massivos (MOOCs). O primeiro contacto com o MOOC inicia-se através dum módulo de ambientação específico desenvolvido de acordo com o modelo pedagógico específico para estes cursos (o modelo europeu sMOOC). Constituindo-se como uma experiência de aprendizagem não-formal e decorrendo em cenários abertos e massivos, as suas características e dinâmicas colocam desafios aos seus designers distintos dos cursos online formais. O estudo que agora se apresenta procurou dar resposta às seguintes questões de investigação:

- Quais as características que um Módulo de Ambientação deve adotar nos MOOCs?

- Será o Módulo de Ambientação capaz de funcionar como um catalisador para o sucesso dos MOOCs?

Tratando-se dum estudo de caso, os dados foram recolhidos através dos seguintes instrumentos: a) aplicação de um questionário aos participantes; b) realização de uma entrevista; c) análise das interações desenvolvidas pelos participantes ao longo da primeira semana, ou seja, o período em que decorreu a ambientação nas plataformas do curso.

Após a análise dos dados recolhidos e tratamento estatístico foi possível concluir que o Módulo de Ambientação concebido foi bem aceite pelos participantes, que o consideraram uma peça-chave, em termos motivacionais e elemento de envolvimento no curso garantindo assim, a sua continuidade e permanência neste MOOC. Além desses fatores foi ainda considerado o seu contributo como ponto de partida para a criação de comunidades de prática no MOOC bem como a sua identificação como ingrediente essencial para a inclusão digital e familiarização com a dinâmica do MOOC e a promoção de códigos de conduta e convivência online. Palavras chave: educação aberta, MOOC, envolvimento, comunidade de aprendizagem
\end{abstract}

\section{Abstract}

This communication intends to analyze the impact of the first days online of the participants in open and massive contexts - Open Online Courses and Massive (MOOCs). The first contact with the MOOC begins with a specific Environment Module (Boot Camp) developed according to the specific pedagogical model for these courses (the European model sMOOC). By forming non-formal learning experiences and taking place in open and massive settings, their characteristics and dynamics pose new challenges, different from the existents in formal online courses. The present study sought to address the following research questions:

- What are the characteristics that the Boot Camp should adopt in MOOCs?

- Will the Boot Camp be able to function as a catalyst for the success of MOOCs?

In this study case, the data were collected through the following instruments: a) application of a questionnaire to the participants; B) conducting an interview; C) analysis of the interactions developed by participants during the first week, that is, the period during which the course platforms were set.

After analysing the collected data and statistical treatment it was possible to conclude that the designed Boot Camp was well accepted by the participants, who considered it a key piece, in motivational terms and as an involvement element in the course, thus ensuring its continuity and permanence in this MOOC. In addition to these factors, their contribution was also considered as the starting point for the creation of communities of practice in the MOOC. In addition to these values, digital inclusion and familiarization with the dynamics of the MOOC and the promotion of codes of conduct and online coexistence were also identified as essential.

Keywords: Learning community, Open education, Involvement, MOOC, BootCamp

\section{Introdução}

A educação tem vindo a sofrer influências diversas de várias origens: sociais, económicas e tecnológicas. A educação a distância, que surgiu no século passado com o uso de tecnologias como o correio, livros, rádio e televisão, atualmente, está diretamente associada a uma panóplia de recursos tecnológicos baseados no computador (Beetham e Sharpe, 2013).

$\mathrm{O}$ ponto fulcral a considerar no que toca à educação aberta é a criação de oportunidades que permitam o acesso à aprendizagem de uma forma igualitária (educação para todos), usando para tal, recursos educacionais abertos que fomentam, em simultâneo, práticas colaborativas.

Assim a educação aberta é definida como um "movimento emergente de educação combina a tradição de partilha de boas ideias com colegas educadores e da cultura da internet, marcada pela colaboração e interatividade. Esta metodologia de educação é 
construída sobre a crença de que todos devem ter a liberdade de usar, personalizar, melhorar e redistribuir os recursos educacionais, sem restrições. Educadores, estudantes e outras pessoas que partilham esta crença estão unindo-se em um esforço mundial para tornar a educação mais acessível e mais eficaz". (Declaração da Cidade do Cabo, 2007).

Neste contexto, os MOOC são uma nova forma de transmissão massiva de conhecimento caracterizada por ser aberta a todos ministrada a distância e recorrendo à internet (Teixeira et. al, 2015).

Face ao seu crescimento abrupto e à visibilidade dos MOOCs, o jornal The New York Times publicou um extenso artigo sobre este fenómeno educativo, o que fez com que 2012 fosse denominado como "o ano dos MOOCs" (Pappano, 2012).

Sendo um tópico ainda emergente, importa efetuar um enquadramento histórico dos MOOCs analisando o seu desenvolvimento e caracterizando diferentes aspetos que lhe são inerentes. Assim, destacam-se as áreas de utilização com maior probabilidade de sucesso para os MOOCs, os diferentes modelos utilizados para estruturar os MOOCs, as principais plataformas já existentes para a persecução de cursos MOOC, os principais promotores deste tipo de cursos, e por fim, identificar os principais desafios, de modo a responder a algumas questões como por exemplo:Quais as principais barreiras ao desenvolvimento dos MOOCs? Qual a perspetiva das universidades e grandes empresas sobre os MOOCs? Qual a perspetiva dos participantes face a estes cursos?

O movimento MOOC, advém da convergência da educação aberta, do software livre e da disponibilização de conteúdos abertos e deu os primeiros passos através do consórcio OpenCourseWare do Massachusetts Institute of Technology (Sanchez-Gordon e Luján-Mora, 2014).

Para aumentar o sucesso dos MOOCs há ainda vários desafios a superar. Como afirma Milman (2012), apesar de se verificarem no seu início um elevado número de inscrições nos MOOC, muitos dos participantes acabam por abandonar os cursos, apontando-se uma taxa de desistência considerável quando comparada com a taxa de desistência relativa a cursos online pagos.

Assim, e indo de encontro a esta nova temática que tem sido alvo de várias reflexões e estudos, esta comunicação pretende assentou na indagação do papel que o Módulo de Ambientação pode assumir neste tipo de curso. $\mathrm{O}$ estudo de caso proposto neste trabalho, centra-se no Módulo de Ambientação dos cursos MOOC integrados no projeto ECO, o modelo sMOOC e visa exprapolar conclusões sobre a importância do mesmo para o sucesso deste tipo de cursos e como podem influenciar a interação e a motivação dos participantes durante o curso.

A este facto não são alheios os próprios fundamentos que estão na génese dos MOOC integrados neste modelo (sMOOC) (Brouns et al., 2016) entre as quais a construção de conhecimento centrado nos participantes, a necessidade de auto-motivação e auto-disciplina dos participantes e a baixa interatividade que caracteriza, em muitos casos, outros modelos de MOOC já desenvolvidos.
Tendo em consideração esta problemática, este Módulo de Ambientação poderá permitir dar a conhecer as características do funcionamento destes MOOCs, das plataformas de aprendizagem no qual se integram e o desenvolvimento duma cultura adequada à educação a distância, permitindo aos participantes interagirem mais facilmente com os facilitadores e organizadores dos cursos e entre a comunidade de participantes. Quais os parâmetros/características que o Módulo de Ambientação deverá abarcar? Será o Módulo de Ambientação capaz de funcionar como um catalisador do sucesso dos MOOCs? Poderá este módulo ser um contributo para várias comunidades dentro do MOOC possam emergir e formar-se uma comunidade para além do curso?

Estas são algumas das questões que emergem do universo MOOC e para as quais este estudo procurou obter respostas.

\section{Estudo de Caso}

O presente estudo teve como foco o Módulo de Ambientação e em que medida é, ou não, um elemento diferenciador no modelo sMOOC, visando recolher as perspetivas dos participantes e posterior análise sobre a importância atribuída ao referido módulo.

O presente estudo tem como base os dados recolhidos nos MOOCs: "Competências Digitais para Professores" e "Lisboa e o Mar: uma História de Chegadas e Partidas" e "Alterações Climáticas: ligando Ciência e Experiências de Vida".

Assim, foram realizados os seguintes instrumentos: Questionário - aplicação dum questionário aos participantes dos MOOCs no final do Módulo de Ambientação; os seus objetivos foram: i) conhecer as expectativas dos participantes dos cursos; ii) avaliar qual o papel do Módulo de Ambientação em geral e no percurso de cada participante; iii) em que medida a frequência deste módulo influenciou a participação e a continuidade dos participantes no MOOC;

Entrevista - uma entrevista aos participantes que frequentaram o Módulo de Ambientação na sua totalidade, para a posteriori possibilitar uma análise mais aprofundada sobre o Módulo de Ambientação e retirar conclusões sobre o seu design instrucional;

Análise das interações (mensagens) - uma análise quantitativa e qualitativa das mensagens publicadas durante todo o período do Módulo de Ambientação de um dos MOOCs: "Competências Digitais para Professores"; análise das mensagens publicadas posteriormente ao Módulo de Ambientação (decorrer do curso) com a hashtag do módulo.

Por fim, analisar todos os dados recolhidos neste estudo que "consistem em observações diretas e indiretas, entrevistas, questionários iniciais e finais (por exemplo inquirir acerca de conhecimentos informáticos), vídeo, documentos (trabalhos dos alunos), relatórios, o "diário de bordo", etc." (Coutinho e Chaves, 2002:224).

Desta análise de dados proveem as conclusões inerentes a este estudo. Todavia, para validar as conclusões retiradas da análise dos dados e comprovar que correspondem fidedignamente à realidade 
educacional vivenciada pelos participantes, foi realizada ums triangulação metodológica, em particular, a triangulação das fontes de dados, em que se confrontaram os dados provenientes de diferentes fontes tais como questionários, entrevista, gravação áudio, observações diretas (Denzin,1970:34).

\section{Resultados obtidos no Questionário}

O questionário é um dos instrumentos mais utilizados para obter informação acerca de uma determinada população, constituindo "um instrumento de medida que traduz os objetivos de um estudo com variáveis mesuráveis. Ajuda a organizar, a normalizar $e$ a controlar os dados, de tal forma que as informações procuradas possam ser colhidas de uma maneira rigorosa" (Fortin, 2003:249)

Assim sendo, após a recolha de opinião da "amostra teste" foi possível fazer pequenas retificações ao questionário para que assim, estivesse em conformidade com o objeto de estudo.

O questionário foi aplicado aos participantes que frequentavam dois MOOCs ${ }^{1}$.

Obtiveram-se 199 respostas, das quais 68 delas estavam parcialmente respondidos (menos de 70\%).

Assim, para a análise dos dados foram considerados apenas 131 respostas, correspondentes ao número de questionários totalmente preenchidos.

\section{Perfil dos Participantes}

Os participantes destes cursos eram maioritariamente do sexo feminino $(68 \%)$, com idades compreendidas entre os 31 e 50 anos ( 50\%). É de salientar a existência de um participante com menos de 21 anos e de vários participantes (6\%) com mais de 60 anos o que demonstra a abrangência destes cursos. O seu local de residência é maioritariamente $(76 \%)$ em Portugal, sendo o Brasil o segundo país que apresenta o mais elevado número de participantes (residualmente residentes de Cabo Verde, Moçambique, São Tomé e Príncipe e outros países da Europa).

Relativamente às habilitações literárias a maioria possui bacharelato/licenciatura, e o mestrado assume o lugar da segunda habilitação literária mais predominante. No que diz respeito às profissões dos participantes, embora com um perfil heterogéneo refira-se que, para mais de metade dos inquiridos $(65 \%)$ está relacionada com o ensino, provavelmente devido aos temas abrangidos nestes MOOCs.

\section{Experiência de MOOCs}

Inquiridos sobre experiência em experiências deste tipo (MOOC), 61\% afirmou que se tratava da primeira vez que participavam num curso MOOC e $39 \%$ já eram reincidentes. Destes, $50 \%$ afirma ter seguido parcialmente o último MOOC em que estiveram inscritos e os restantes $50 \%$ afirma ter acompanhado na totalidade o último MOOC frequentado.

\section{Papel do Módulo de Ambientação}

Da opinião dos inquiridos pode-se concluir que a Ambientação "foi um momento de contacto e alguma descoberta", permitindo "ter uma melhor noção do processo de gamificação, bem como conhecer um pouco dos outros participantes", dando "significado aos propósitos e rumo aos objetivos", funcionando "como uma espécie de aquecimento preparação e avaliação do ambiente", visto que "os moocs são bastante caóticos, a ambientação ajuda a compreender”.

Um outro aspeto que sobressai destes MOOC, principalmente em relação ao feedback a Ambientação "Serviu ainda para troca de informação (documentação) sobre a temática". Citando um inquirido, que considera "que nenhum curso online deve ser realizado sem ter um momento de ambientação prévio que permita não só a aquisição de competências relacionadas com as plataformas usadas, mas também o intercâmbio entre os participantes, a fim de facilitar a integração de quem se encontra em dificuldade por ser a primeira vez que realiza um curso destes e ainda permitir que os participantes se conheçam" pode-se perceber uma outra dimensão importante da Ambientação que é que "numa modalidade de formação a distância a componente ambientação permite um maior equilíbrio e igualdade entre os participantes e permite perder alguns dos receios inerentes". Esta vertente, é evidenciada pelo testemunho de um dos inquiridos que refere que a Ambientação permitiu "adquirir competências digitais que julgava não conseguir obter". É ainda importante destacar o papel dos facilitadores e dar os "Parabéns à equipe, mas quero destacar o papel excelente que a professora M... teve".

Em suma, o Módulo de Ambientação como sendo " $A$ alavanca de motivação".

\section{Resultados obtidos nas Entrevistas}

$\mathrm{O}$ inquérito por Entrevista foi uma das técnicas escolhidas para este estudo de caso pois era essencial ter a perceção real sobre a experiência educativa dos participantes, visto que uma entrevista "é utilizada para recolher dados descritivos na linguagem do próprio sujeito, permitindo ao investigador desenvolver intuitivamente uma ideia sobre a maneira como os sujeitos interpretam aspetos do mundo" (Bogdan e Biklen, 1994:134). " A entrevista é um método de recolha de informações que consiste em conversas orais, individuais ou em grupos, com várias pessoas selecionadas cuidadosamente, cujo grau de pertinência, validade e fiabilidade é analisado na perspetiva dos objetivos da recolha de informações". (De Ketele e Rogiers, 1999: 18).

A entrevista teve como objetivos a) conhecer as perceções dos participantes do MOOC sobre a semana de Ambientação; b) avaliar qual o papel da semana de Ambientação no desempenho dos participantes no MOOC.

Foram efetuadas seis entrevistas aos participantes de acordo com um guião previamente elaborado. As

\footnotetext{
1 "Lisboa e o Mar: uma História de Chegadas e Partidas" e "Alterações Climáticas: ligando Ciência e Experiências de Vida" na plataforma EMMA
} 
entrevistas decorreram entre 8 e 23 de Abril tendo sido realizadas através da ferramenta Skype.

\section{Relação entre Ambientação e desempenho}

Relativamente à relação estabelecida entre a semana de Ambientação e o desempenho ao longo do MOOC, os entrevistados consideraram que estão diretamente relacionados. O Módulo de Ambientação promove o ganho de motivação, promove o à-vontade com a plataforma sendo indispensável para "ter um melhor desenvolvimento e sucesso nas várias atividades que se propõe ao longo do curso", funcionando como um elemento tranquilizador pois permite explorar e clarificar as dúvidas iniciais que vão surgindo, sendo "um elemento fundamental para se querer ou não ficar no MOOC e dar continuidade ao projeto", criando uma "vontade de ... manter online e de ... manter naquela atividade até ao limite que fosse possível. Em suma, "a semana de ambientação é tudo".

\section{Relação entre Ambientação e sucesso}

No que respeita ao Módulo de Ambientação enquanto elemento determinante do sucesso do curso MOOC, os entrevistados referem que este assume um papel deveras importante, "uma peça fundamental". Contudo não se trata de um elemento exclusivo, uma vez que este não "determina o sucesso, mas que promove o sucesso", podendo "determinar o sucesso, do ponto de vista de criar esse sentimento de pertença a uma determinada... a um determinado grupo que está a refletir e a construir sobre determinada temática". Assim sendo, pode-se referir que o Módulo de Ambientação assume um papel importante para se ser bem-sucedido num curso MOOC, funcionando como "um fio condutor".

\section{Avaliação da Ambientação}

Quando foi pedido aos entrevistados um balanço do Módulo de Ambientação pode-se constatar que ele foi francamente positivo. Na sua opinião a facilitação desenvolvida pela equipa de facilitação assumiu um papel fundamental contribuindo para a satisfação dos participantes durante este módulo. Foi destacada ainda a componente da interação desenvolvida ao longo deste período e que o Módulo de Ambientação fez com que os participantes se familiarizassem com a plataforma e as funcionalidades que a mesma possui.

Relativamente aos pontos menos positivos, os entrevistados referiram que a Gamificação necessitava de ser mais contextualizada durante o módulo já que a "Gamificação fez com que algumas pessoas se despistassem na utilidade do Módulo de Ambientação". Outro dos elementos referido foi o volume de informação inerente ao carácter massivo dos MOOCs, embora tenham sido transmitidas as "informações necessárias". $\mathrm{Na}$ globalidade, o módulo foi considerado pelos entrevistados como fundamental e referido como: " $E$ necessário, é extremamente necessário o módulo de ambientação. Perfeito, deixar estar do jeito que está" e que "o Módulo de Ambientação é a peça chave para depois o MOOC'.

\section{Resultados obtidos na Análise das Interações}

A observação é uma técnica que privilegia o papel do investigador e que permite que este possa acompanhar de perto o objeto de estudo "descrevendo os comportamentos em seu ambiente [...] extraindo as estruturas reveladoras de significado do fenômeno estudado" (Chizzotti, 2008: 65-76).

A finalidade desta técnica foi ficar a conhecer de uma forma verosímil como as interações se dão entre os participantes e, simultaneamente, conseguir extrair ilações sobre o Módulo de Ambientação, cingindo-se apenas à recolha de dados provenientes da interação. Tal como refere Serrano (2004:32) o que interessa é ficar a "conhecer as realidades concretas nas suas dimensões reais e temporais, o aqui e o agora no seu contexto social".

Assim sendo, esta análise implicou a criação de categorias de acordo com a natureza da participação, tendo sido de novo utilizado o "método comparativo constante" para "categorizar" as mensagens quanto ao seu teor e assim, as poder classificar e, posteriormente, fazer a análise dos dados obtidos.

No caso desta investigação em particular, a análise de conteúdo foi baseada nas mensagens trocadas entre os participantes (participante/participante, participante/ equipa facilitação) ao longo do Módulo de Ambientação ou, a posteriori (mensagens com a hashtag da Ambientação) mas referentes ainda ao módulo em questão.

As interações recolhidas referem-se à participação nas atividades através dos diferentes dispositivos digitais do ambiente virtual do MOOC - Curtas, Blogs, Bookmarks e Partilha de Ficheiros - através dos posts e comentários de toda a comunidade (participantes e equipa de facilitação). A análise de conteúdo focou-se nas mensagens trocadas entre participante/participante e participante/equipa facilitação) ao longo do Módulo de Ambientação ou a posteriori (mensagens com a hashtag da Ambientação) mas, ainda referentes ao módulo. Estas intervenções, depois de coligidas, o seu conteúdo foi classificadocom base no Quadro de Indicadores de Comunidade (Community Indicators Framework, CIF) proposto em Galley, Conole e Alevizou (2012:379).

\section{Conclusões}

Com este estudo de caso evidencia-se que o Módulo de Ambientação assume um papel relevante, num curso online, mas que num curso de natureza massiva, como é o caso dos MOOC, torna-se imprescindível e que funciona como catalisador para uma intervenção mais ativa, consciente e dinâmica dos participantes. Relativamente à contribuição do Módulo de Ambientação para a redução da taxa de desistência podese concluir que pode ser um fator importante, mas não completamente determinante. Este aspeto prende-se com o facto de ser necessário manter os participantes motivados, interessados e envolvidos na criação de conhecimento durante toda a duração do MOOC.

Com base nos dados recolhidos é possível afirmar que o Módulo de Ambientação é fundamental para que os participantes possam ir mais além na sua experiência 
educativa, ganhando e tomando consciência das características do curso, das funcionalidades e potencialidades da plataforma, do desenvolvimento das suas competências digitais necessárias para ter possibilidade de interagir, socializar e desenvolver as atividades propostas ao longo do curso, como também criar e desenvolver conexões entre os demais participantes e desenvolver as suas redes sociais, podendo ainda dar origem à criação de comunidades de prática (Wenger, 1998) e à criação do conhecimento, sem esquecer a assimilação das regras de etiqueta online e do desenvolvimento de sentido crítico aprimorado.

\section{Referências}

Beetham, H., Sharpe, R. (2013). Rethinking pedagogy for a digital age: Designing for 21st century learning. New York: Routledge.

Bogdan, R., Biklen, S., (1994). Investigação Qualitativa em Educação - uma introdução à teoria e aos métodos. Porto: Porto Editora.

Brouns F., Teixeira A., Morgado L., Fano S., Fueyo A., Jansen D. (2017). Designing Massive Open Online Learning Processes: The sMOOC Pedagogical Framework. In: Jemni M., Kinshuk, Khribi M. (eds) Open Education: from OERs to MOOCs. Lecture Notes in Educational Technology. Springer, Berlin, Heidelberg doi: 10.1007/978-3-662-52925-6_16

Chizzotti A. (2008).Pesquisa qualitativa em ciências humanas e sociais. Rio de Janeiro: Vozes.

Coutinho, C., e Chaves, J. (2002). O estudo de caso na investigação em Tecnologia Educativa em Portugal. Revista Portuguesa de Educação, 15(1), 221-244. CIEd: Universidade do Minho

De Ketele, J., e Rogiers, X. (1999). Metodologia da Recolha de Dados. Fundamentos dos Métodos de Observações, de Questionários, de Entrevistas e de Estudo de Documentos. Lisboa: Instituto Piaget.

Declaração da cidade do Cabo (2007). Declaração da cidade do cabo para a Educação Aberta: Abrindo a promessa de Recursos Educacionais Abertos.

Denzin, N. (1970). The Research Act: A Theoretical Introduction to Sociological Methods, Englewood Cliffs, NJ: Prentice Hall.

Fortin, M. (2003). O Processo de Investigação. Da Concepção à realização. Loures, Portugal: Lusociência.

Galley, R., Conole, G., e Alevizou, P. (2012). Community indicators: a framework for observing and supporting community activity on Cloudworks. Interactive Learning Environments, 22(3), 373-395.

Milman, B. (2012). MOOCs What are day? Plus 20 Questions We Should Be Asked About Them. Distance Learning, 9(4), 91-93.

Teixeira, A., Mota, J., Morgado, L., \& Spilker, M. (2015). iMOOC: Um Modelo Pedagógico Institucional para Cursos Abertos Massivos Online (MOOCs). EducaçãO, Formação \& Tecnologias, ISSN 1646933X, 8(1), 4-12.

Pappano, L. (2012). The year of the MOOC. The New York Times, 02 Nov.
Sanchez-Gordon, S., \& Luján-Mora, S. (2014). MOOCs gone wild. in Proceedings of the 8th International Technology, Education and Development Conference (INTED 2014), 1449-1458, Valencia, Spain.

Serrano, G. (2004). Investigación cualitativa. Retos $e$ interrogantes, I. Métodos. Madrid: Ed. La Muralla.

Wenger, E. (1998), Communities of Practice, Cambridge University Press. 